\title{
Articaine use does not routinely eliminate the need for palatal injections for primary maxillary molar extractions: a randomized cross-over clinical trial
}

\author{
Zahra Bahrololoomi ${ }^{1} \cdot$ Nahid Maghsoudi $^{1}$ (i)
}

Received: 1 October 2020 / Accepted: 15 November 2021 / Published online: 1 December 2021

(c) The Author(s), under exclusive licence to Springer-Verlag GmbH Germany, part of Springer Nature 2021

\begin{abstract}
Purpose The aim of this study was to compare single buccal infiltration of $4 \%$ articaine versus routine buccal and palatal injections of $2 \%$ lidocaine during bilateral extraction of maxillary primary molars.

Methods Thirty healthy children aged 6-9 years old requiring bilateral extraction of maxillary primary molars were enrolled into the trial. Randomization was performed in two steps. Routine buccal and palatal infiltrations were administered using $2 \%$ lidocaine in the control side. Four percent articaine was used for single buccal infiltration in the intervention side. WBFP scale (Wong-Baker FACES Pain scale) was employed for subjective assessment of pain. For objective evaluation of pain, FLACC scale (Face, Legs, Activity, Cry, Consolability) and physiological parameters of blood pressure and pulse rate were recorded. Results Analyses did not show any significant differences in blood pressure, pulse rate, and FLACC scale between groups $(P$ value $>0.05)$. However, statistically lower WBFP scores were observed in articaine group as compared to lidocaine group $(P$ value $<0.05)$; the difference was less than one unit which is not clinically considerable.

Conclusion Single buccal infiltration of $4 \%$ articaine can be administered as an alternative for conventional infiltration of $2 \%$ lidocaine to avoid fear or uncooperative behavior of the child patient. However, the assessment of palatal tissue anesthesia is quite necessary before the procedure is initiated. In case of failure in achieving palatal anesthesia, a palatal injection should be given.
\end{abstract}

Keywords Articaine $\cdot$ Lidocaine $\cdot$ Local anesthesia $\cdot$ Tooth extraction $\cdot$ Children

\section{Introduction}

Tooth extraction is one of the most aggressive treatments in pediatric dentistry. Except in emergencies, it is postponed to the last stage of treatment plan. Local anesthesia is commonly used for pain control during tooth extraction. Common technique of local anesthesia for maxillary tooth extraction is buccal and palatal infiltrations of $2 \%$ lidocaine. Lidocaine is the gold standard among anesthetic agents [1]. A deep local anesthesia helps the dentist to guide the behavior and reduce anxiety and disruptive behaviors in children. Nevertheless, local anesthesia injection is a serious

Nahid Maghsoudi

nahidmaghsoodi@yahoo.com

1 Department of Pediatric Dentistry, Social Determinants of Oral Health Research Center, Dentistry Faculty, Shahid Sadoughi University of Medical Sciences, Yazd, Iran challenge in pediatric patients. Topical anesthetic gel is applied on dried mucosa before injection to minimize needle penetration pain and sting. However, injection in some areas including palatal mucosa remains extremely painful [2-4]. High density of palatal submucosa and its tight attachment to the underlying mucoperiosteum cause intense pressure to the surrounding tissue during and after injection. Dense neural innervations of the palate will exacerbate the pain perception [1]. Experiencing such pain can lead to non-cooperation and misbehavior, high anxiety or fear in the child, and even loss of the child's trust in the dentist [2-4].

Multiple techniques have been introduced to eliminate pain and discomfort of pediatric patients during local anesthesia injection, including cooling the place of injection, computerized injection system, using pressure during injection, and distraction, but none of these methods provides a painless injection specially into the palatal region; however, the results of the studies are very different and there is no comprehensive agreement on the best technique [5-7]. 
A newly proposed technique is single buccal infiltration of $4 \%$ articaine without any palatal injection. Articaine is a new amide local anesthesia agent presented in 1969. It is 1.5 times more potent than lidocaine. Unlike other amide anesthetics, articaine has a thiophene ring instead of benzene ring; thiophene ring boosts tissue penetration of articaine. Thus, application of single buccal infiltration of articaine in maxilla can provide efficient anesthesia in both buccal and palatal tissues; this is due to good penetration of articaine through soft and hard tissues and lower bone density in children $[2,8,9]$. It is metabolized in serum (by esterase) and liver (by microsomal enzymes). It has a shorter longevity than other amide anesthetic agents resulting in less toxicity potential. Articaine is available in the market in a $4 \%$ concentration with 1:100,000 or 1:200,000 epinephrine [8].

A potential side effect of articaine is methemoglobinemia; however, so far there has been no report in common dental treatments. In addition, there have been reports of inferior alveolar nerve paresthesia following administration of articaine block; but there has been no reports indicating complications following infiltration of articaine in mandible or maxilla [10, 11].

Due to the disadvantages of buccal and palatal infiltrations of lidocaine in the maxilla and the advantages of single buccal infiltration of articaine, several studies have been performed to compare the efficacy of these two anesthetics during maxillary primary molars extraction. As yet, studies have not achieved a comprehensive agreement on efficacy of articaine and lidocaine during maxillary primary molars extraction [12-20]. None of the previous studies has equalized the confounding factors which have direct effect on the perception of pain such as child's personality and temperament, emotional development, family, and culture. Moreover, available evidence has low quality with high risk of bias and methodological inconsistencies. More accurate methodologies which provide less heterogeneous data for meta-analysis are needed [21].

The objective of this manuscript was to compare single buccal infiltration of $4 \%$ articaine versus routine buccal and palatal injections of $2 \%$ lidocaine during bilateral extraction of maxillary primary molars. The study hypothesis was equivalent anesthesia depth in both agents.

\section{Material and methods}

\section{Participants and study design}

This double-blind cross-over randomized clinical trial was performed on the children aged 6 to 9 years old who referred to pediatric dental clinic of Faculty of Dentistry of Shahid Sadoughi University of Medical Sciences. The study protocol was approved and supervised by the Ethics Committee of
Shahid Sadoughi University of Medical Sciences in accordance with the declaration of Helsinki (DoH). The trial was registered at Iran Registration Clinical Trial Center with an online registration number: IRCT20191002044953N1.

Parents fulfilled the informed consent after receiving a complete description of the study process. Parents were informed about the conditions which result in patient exclusion; possible conditions were child's non-cooperation, diagnosis of acute abscess, etc. Even with exclusion of the patients, they received adequate treatments.

\section{Inclusion criteria}

- Healthy children aged 6 to 9 years old without learning disabilities or allergy to drugs or local anesthetic agents

- Requiring bilateral extraction of primary maxillary molars with at least one third of root length

- Groups 3 or 4 of Frankl behavioral rating scale

- Capable of communication in Farsi

\section{Exclusion criteria}

- Children showing uncooperative behavior during treatment

- Acute abscess

- Use of analgesics before treatment

\section{Interventions}

Medical and dental histories were taken at the first examination visit. The maxillary molars were examined clinically and radiographically, and the child's behavior was assessed by prophylaxis and fluoride therapy. Children meeting inclusion criteria whose parents signed the informed consent entered into the trial.

After inclusion into the trial, each patient was randomly assigned into one of the two groups. Lidocaine in group 1 and articaine in group 2 were injected for the local anesthesia at the first appointment; at the second visit, this process was reversed. After that a blocked random list was used for determination of which side of the maxilla to be treated first (which side will be test side and which will be control side). Thus, one of these 4 cases was possible for each patient.

- Extraction of right molar with buccal and palatal infiltration of lidocaine at the first visit

- Extraction of right molar with buccal infiltration of articaine at the first visit

- Extraction of left molar with buccal and palatal infiltration of lidocaine at the first visit

- Extraction of left molar with buccal infiltration of articaine at the first visit. 
The contralateral molar was extracted at the second visit with the other anesthetic agent.

For topical anesthesia, 20\% benzocaine gel (Master-Dent, Dentonics Inc, Tophill road, Monroe, USA) was applied on dried mucosa for $1 \mathrm{~min}$. For buccal infiltration, $1.8 \mathrm{ml}$ of anesthesia solution was injected between buccal roots of primary molar with a slow rate of $1 \mathrm{ml}$ per min. The 27 -guage short needle (SHINjet, Shinhung, Seoul, Korea) penetrated into the tissue $2-3 \mathrm{~mm}$ aligned along the long axis of the tooth.

In the control group, routine buccal and palatal infiltrations were administered using 2\% lidocaine with 1:100,000 epinephrine (Persocaine-E, Darou Pakhsh, Tehran, Iran). In this group, benzocaine gel was simultaneously administered in buccal and palatal mucosa before injection.

Four percent articaine with 1:100,000 epinephrine (Dentacain 200, Exir Pharmaceutical Co, Borujerd, Iran) was used for single buccal infiltration in the intervention group. After 10 min waiting, complete anesthesia of buccal and palatal tissues of the tooth was assessed using an explorer and after that by observing the patient's reactions during extraction. If the child reported pain during anesthesia assessment using the explorer or during tooth extraction, a supplemental injection was administered. After obtaining adequate anesthesia, the tooth was extracted. Positive reinforcement, tell-showdo, nonverbal behavior guidance, and verbal distraction were used as behavioral guidance techniques during anesthesia and tooth extraction at both visits.

Wong-Baker FACES Pain scale was used to subjectively assess pain immediately after extraction. This self-report scale had been described for the patient before the anesthesia administration. It comprises six cartoon faces ranging from a smiling "no hurt" face to a crying "hurts worst" pain.

Fig. 1 Wong-Baker FACES Pain scale
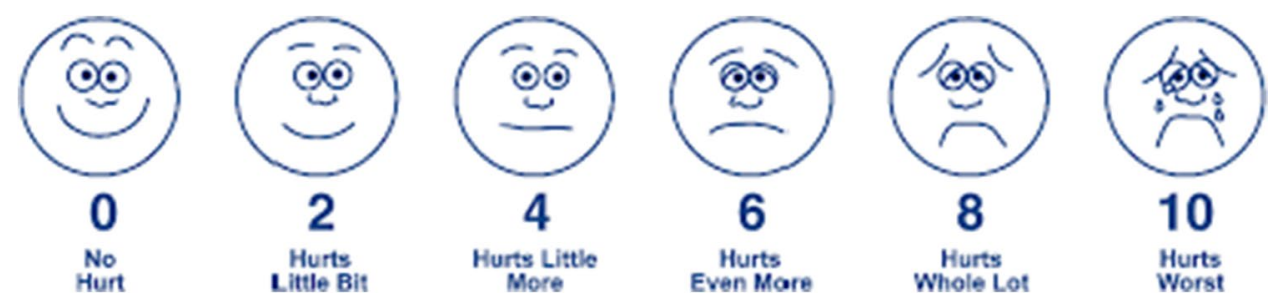

Each face was assigned a number ranging from 0 to 10 . This parameter was considered as the primary outcome (Fig. 1).

For objective evaluation of pain, FLACC scale (Face, Legs, Activity, Cry, Consolability) and physiological parameters of blood pressure and pulse rate were used. FLACC scale consists of five parameters: (1) facial expression, (2) legs movement, (3) activity, (4) cry, and (5) consolability (Fig. 2).

Hemodynamic indicators were recorded by a digital blood pressure monitor (Omron M6 Comfort Blood Pressure Monitor, Omron Corp, Kyoto, Japan) and a pulse oximeter (Beurer PO30 Pulse Oximeter, Beurer GmbH, Soflinge Str, Germany) which was attached to the right index finger before anesthesia administration and immediately after extraction.

\section{Sample size determination}

Based on a confidence level of $95 \%$, power of $80 \%$, and standard deviation of 2.8 for pain score (WBFP as primary outcome) and considering two units of difference in pain score change between two groups, the size of each group was calculated 30 . Thus, the total sample size was 60 teeth.

\section{Randomization and random allocation concealment}

Participants were grouped using two random 30-item computer-generated lists prepared by a statistician, who was not involved in patient recruitment or assessments. The first list was generated using simple randomization method; this list determined the anesthetic drug of first visit (A, lidocaine or $\mathrm{B}$, articaine). The second list was created using block randomization method with a block size of two (AB or $\mathrm{BA}$ );
Fig. 2 Face, Legs, Activity, Cry, Consolability

\begin{tabular}{|c|c|c|}
\hline Category & Description & Score \\
\hline \multirow[t]{3}{*}{ Face } & $0=$ No particular expression or smile & 0 \\
\hline & 1 = Occasional grimace $/$ frown withdrawn or disinterested & 1 \\
\hline & $2=$ Frequent $/$ constant quivering chin, clenched jaw & 2 \\
\hline \multirow[t]{3}{*}{ Legs } & $0=$ Normal position or relaxed & 0 \\
\hline & $1=$ Uneasy, restless, tense & 1 \\
\hline & $2=$ Kicking or legs drawn up & 2 \\
\hline \multirow[t]{3}{*}{ Activity } & $0=$ Lying quietly, normal position, moves easily & 0 \\
\hline & $1=$ Squirming, shifting back and forth, tense & 1 \\
\hline & $2=$ Arched, rigid or jerking & 2 \\
\hline \multirow[t]{3}{*}{ Cry } & $0=$ No cry & 0 \\
\hline & $1=$ Moans or whimpers, occasional complaint & 1 \\
\hline & 2 = Crying steadily, screams or sobs, frequent complaints & 2 \\
\hline \multirow[t]{3}{*}{ Consolability } & $0=$ Content and relaxed & 0 \\
\hline & $1=$ Reassured by occasional touching, hugging or being talked to, distractable & 1 \\
\hline & $2=$ Difficult to console or comfort & 2 \\
\hline
\end{tabular}


in fact, the randomization was done within each block. It determined which side of the maxilla will be the test side and which will be the control side (A, right molar or B, left molar). For allocation concealment, the sequences of both lists were written separately on cards and placed in opaque and sealed envelopes. The envelopes were put into two separate boxes. The patient chose one envelope of each box.

\section{Blinding}

The investigator who performed the procedures was not blinded; thus, another investigator collected the data. The patients were not aware of intervention type; therefore, this study is double-blinded.

\section{Statistical analysis}

Data analysis was conducted using SPSS 22 (SPSS INC, Chicago IL, USA). Intergroup comparisons of pulse rate and blood pressure were done using independent sample $T$ test, and intragroup comparisons of pulse rate and blood pressure at two different time intervals were performed using paired $T$ test. Intergroup comparisons of WBFPS and FLACC were done by chi-square and Mann-Whitney tests.

\section{Results}

\section{Participant characteristics}

Thirty children aged 6 to 9 years old, who referred to pediatric dentistry clinic of Shahid Sadoughi University of Medical Sciences, were enrolled in the research between January 2020 and June 2020. Initially, 32 children were eligible to participate in the study; however, parents of two children declined to participate. Finally, 28 children completed the trial. Two children did not attend the second visit, and their parents did not respond any phone calls (Fig. 3). Of the total participants, 15 (53\%) were girls and 13 (46\%) were boys. The mean age was $7.45 \pm 0.85$ years old. Of the 56 maxillary primary molars, 25 were first molars and 31 were second molars. Both of the extracted molars were the same in all cases except three of them; in these three cases, one of the teeth was the first molar and another was the second. There were $12(42 \%)$ first molars and 16 (57\%) second molars in the lidocaine group. The articaine group consisted of 13 (46\%) first molars and $15(53 \%)$ second molars.

\section{Clinical parameters}

In the lidocaine group, the mean systolic and diastolic blood pressures before anesthesia injection were 103.11 \pm 14.642 and $71.86 \pm 11.594$, respectively. The mean systolic and diastolic blood pressures before anesthesia in the articaine group were $101.46 \pm 14.185$ and $68.14 \pm 8.822$, respectively. Post-extraction mean systolic and diastolic blood pressures for the lidocaine group were $115.86 \pm 16.852$ and $78.86 \pm 16.429$, respectively. These parameters were $111.82 \pm 13.974$ and $76.32 \pm 10.335$ in the articaine group. Post-extraction systolic blood pressure had the maximum difference between the groups. Independent sample $t$ test showed no statistically significant difference in before injection or post-extraction blood pressure between groups $(P$ value $>0.05)$ (Table 1).

The mean pulse rate before injection and post extraction in the lidocaine group were $89.50 \pm 12.048$ and $99.93 \pm 11.972$, respectively. These outcomes were $89.54 \pm 10.330$ and $98.39 \pm 11.458$ in the articaine group. There was no significant difference in pulse rate between groups $(P$ value $>0.05)$ (Table 1$)$.

Score 0 was the most prevalent score $(35.7 \%)$ in objective evaluation of pain using FLACC in the lidocaine group. The prevalence of score 1 and 2 were $28.6 \%$ and $21.4 \%$, respectively. The most frequent score of articaine group was $2(42.9 \%)$; after that score $1(32.1 \%)$ and score $0(17.9 \%)$ were seen more frequent than others.

The mean values of FLACC scale were 1.18 in the lidocaine group and 1.39 in the articaine group. Based on Pearson chi-square test, there were no statistically significant differences between the two groups $(P$ value $=0.295)$ (Table 2$)$.

Score 2 was the most frequent score $(53.6 \%)$ in subjective evaluation of pain and anxiety using WBFPS; after that score $4(32.1 \%)$ and score $0(14.3 \%)$ had the most prevalence in lidocaine group. Score $2(50.0 \%), 0$ (39.3\%), and 4 $(10.7 \%)$ of WBFP had the most frequency in the articaine group, respectively.

The mean score of WBFPS was 2.36 and 1.43 in the lidocaine and articaine group, respectively. Chi-square showed meaningful differences between groups $(P$ value $=0.037)($ Table 3$)$.

Mann-Whitney test did not show any statistically significant differences in FLACC scores between the two groups $(P$ value $=0.289)$. However, there was statistically considerable differences in WBFP scores between the two groups $(P$ value $=0.013$ ).

No side effects were reported following lidocaine and articaine administration or tooth extraction.

\section{Discussion}

The aim of this split-mouth double-blind clinical trial was to compare $2 \%$ lidocaine and $4 \%$ articaine during primary maxillary molars extraction. There is not any similar clinical trial with a cross-over design comparing single buccal infiltration of articaine and conventional buccal and palatal injections of lidocaine for primary molars extraction. Each patient needed 


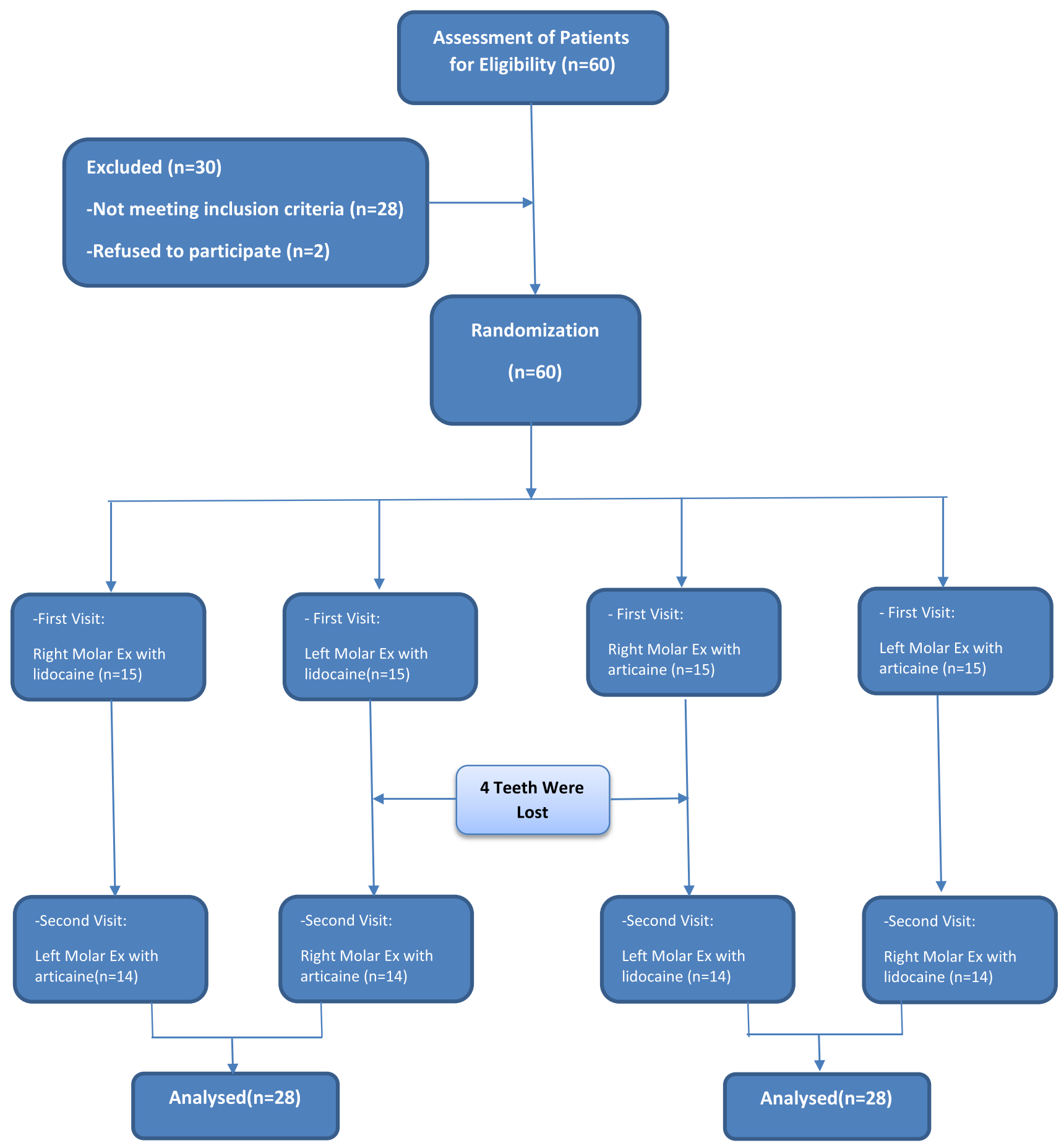

Fig. 3 Flow chart of the trial

bilateral extraction, and samples were randomized with two random lists. These two steps of randomization and random allocation concealment of participants minimize the selection bias. Blinded dentist assessed and recorded the outcomes, children were not aware of interventions type, and this design reduces ascertainment and information biases.
The outcomes were evaluated by the objective and subjective assessment tools. Blood pressure, pulse rate, and FLACC scale assessed anesthesia depth objectively and WBFP scale measured the pain and anxiety subjectively. These indicators examined pain from several different dimensions, including behavioral and physiological, which 
Table 1 Blood pressure and pulse rate

\begin{tabular}{lllllll}
\hline & Group & N & Mean & Std. deviation & Mean difference & Sig. \\
\hline Systolic blood pressure, before injection & Lidocaine & 28 & 103.11 & 14.642 & 1.643 & 0.672 \\
& Articaine & 28 & 101.46 & 14.185 & & \\
Systolic blood pressure, post extraction & Lidocaine & 28 & 115.86 & 16.852 & 4.036 & 0.334 \\
& Articaine & 28 & 111.82 & 13.974 & & \\
Diastolic blood pressure, before injection & Lidocaine & 28 & 71.86 & 11.594 & 3.714 & 0.183 \\
& Articaine & 28 & 68.14 & 8.822 & & \\
Diastolic blood pressure, post extraction & Lidocaine & 28 & 78.86 & 16.429 & 2.536 & 0.492 \\
& Articaine & 28 & 76.32 & 10.335 & & \\
Pulse rate, before injection & Lidocaine & 28 & 89.50 & 12.048 & -0.036 & 0.991 \\
& Articaine & 28 & 89.54 & 11.972 & & \multirow{2}{*}{0.626} \\
Pulse rate, post extraction & Lidocaine & 28 & 99.93 & 10.330 & 1.536 & \\
& Articaine & 28 & 98.39 & 11.458 & & \\
\hline
\end{tabular}

\begin{tabular}{|c|c|c|c|c|c|c|}
\hline & \multicolumn{2}{|l|}{ Total } & \multicolumn{2}{|c|}{ Lidocaine } & \multicolumn{2}{|c|}{ Articaine } \\
\hline & Count & $\%$ within group & Count & $\%$ within group & Count & $\%$ within group \\
\hline 0 & 15 & $26.8 \%$ & 10 & $35.7 \%$ & 5 & $17.9 \%$ \\
\hline 1 & 17 & $30.4 \%$ & 8 & $28.6 \%$ & 9 & $32.1 \%$ \\
\hline 2 & 18 & $32.1 \%$ & 6 & $21.4 \%$ & 12 & $42.9 \%$ \\
\hline 3 & 5 & $8.9 \%$ & 3 & $10.7 \%$ & 2 & $7.1 \%$ \\
\hline 4 & 1 & $1.8 \%$ & 1 & $3.6 \%$ & 0 & $0.0 \%$ \\
\hline Total & 56 & $100.0 \%$ & 28 & $100.0 \%$ & 28 & $100.0 \%$ \\
\hline
\end{tabular}

Table 3 Wong-Baker FACES Pain scale

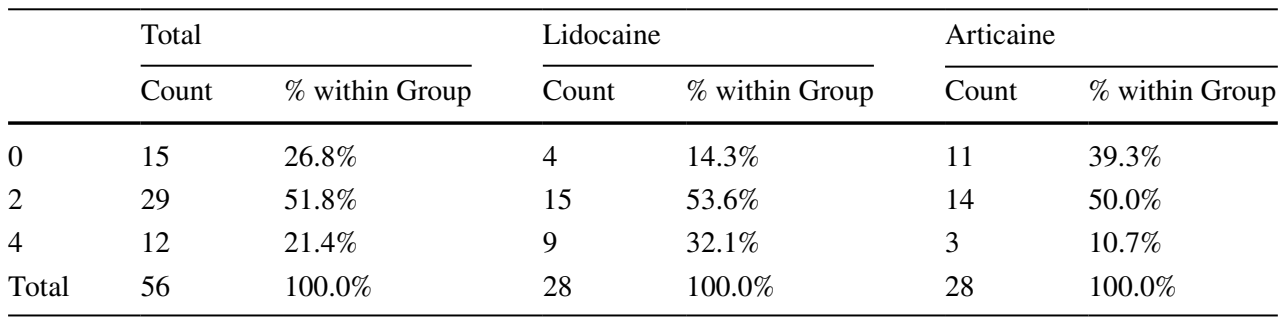

leads to a multidimensional evaluation of the pain experience and provides more comprehensive results [22].

Psychological, familial, social, cultural and genetic factors can influence on the experience of pain. Split-mouth design of this study and performing only one type of dental treatment in all samples eliminate such interfering factors.

Hemodynamic parameters did not show the superiority of the drugs over each other. The average values of postextraction blood pressure and pulse rate were greater in the lidocaine group; however, these differences were not statistically significant $(P$ value $>0.05)$. These results are in accordance with previous studies $[13,15,16,19,20]$. Mittal et al. compared the anesthetic efficacy of articaine and lidocaine during primary maxillary molar extraction; however, Malamed et al. and Arrow et al. compared buccal infiltration of $4 \%$ articaine and mandibular block of $2 \%$ lidocaine. The samples of a study by Ram and Amir were
40 maxillary and 20 mandibular teeth. The majority of performed treatments were simple $[13,15,16,19,20]$. The design of the study by Sharma et al. was almost similar to the present study. They compared single buccal infiltration of articaine and lidocaine during bilateral extraction of maxillary premolars. Similar to our study, there were not any significant differences in blood pressure and pulse rate between groups [18]. A study by Bahrololoomi et al. showed different results. In Bahrololoomi study, the efficiency of $2 \%$ lidocaine mandibular block and $4 \%$ articaine infiltration during pulpotomy of primary mandibular second molars were evaluated. Pulse rate, blood pressure, respiratory rate, and oxygen saturation were higher in the lidocaine group; however, this difference was significant only for pulse rate and respiratory rate. Different results are related to the different methodologies of the two studies [14]. 
FLACC scale as a behavioral and observational scale possesses good internal consistency and adequate reliability and validity [23]. The mean FLACC in the articaine group was higher than lidocaine group, but the difference was not statistically significant $(P$ value $>0.05)$. This is in agreement with the results of Koli et al. study [20].

Different pharmacological factors can influence on local anesthetic drugs' efficacy. One of these factors is the ability of tissue diffusion which is related to molecular configuration and partition coefficient. Articaine has a thiophene ring instead of benzene ring resulting in more tissue diffusion compared to lidocaine. Generally, thiophene derivates block cellular channels in lower concentrations than benzene derivates. In addition, sodium channels are blocked by articaine at lower concentrations than potassium channels. These properties might increase articaine efficacy. However, there were not any significant differences between groups in the present study and the other researches. This could be due to higher partition coefficient of lidocaine and higher affinity of articaine for binding to plasma proteins. In fact, partition coefficient describes lipophilicity or fat solubility, since the cellular membrane is mainly made of phospholipids, it will be an important part of drug's function. Fat solubility of articaine and lidocaine are 1.5 and 4.0, respectively. This might justify similar clinical efficacy of articaine and lidocaine $[8,24]$.

Self-report measurement of pain is the recommended method in the literatures. WBFP scale is a reliable and valid method of pain assessment, it is available, free of charge, easy, and quick, it requires minimal instructions, and all age groups and nurses prefer it to other assessment methods [25]. In this research, the mean of WBFP score was higher in lidocaine group and the differences were statistically considerable $(P$ value $<0.05)$. This is in agreement with Ghadimi et al., Alzahrani et al., and Ram and Amir and Mittal et al.; however, the reported differences in these studies were not statistically significant $[12,16,19,26]$. VAS is another self-report scale of pain assessment. Sandilya et al. and Bataineh et al. did not report any significant differences in VAS between groups. They conducted a cross-over clinical trial for bilateral extraction of maxillary premolars [17] and Bataineh et al. compared the efficacy of articaine without a palatal injection during tooth extraction in the anterior and posterior region of maxilla [27].

The intense pressure during tooth extraction can be difficult to differentiate from pain, especially for pediatric patients. The possible pressure sensation was explained for child before the procedure was initiated using tell-show-do technique. However, it is occasionally not possible to differentiate the sense of pressure from pain, even for adult patients, and it is possible some levels of exaggerated scores of WBFPS were being reported in the present study. However, objective pain assessment and the split-mouth design of the trial may modulate the impact of such confounding factor on the overall results.

In this study, 1 tooth of articaine group and 2 teeth of lidocaine group needed supplemental injections; in these cases, the tooth was extracted after additional injection. Five out of these seven teeth were second molars; high thickness of zygomatic process overlying the molar roots can interfere with drug diffusion. The number of supplemental injections are considerably smaller than one reported by Mittal et al. They observed complete palatal anesthesia in only one case of articaine group [19]. Alzahrani et al,. reported two cases of articaine group and one case of lidocaine group requiring additional injections [12]. Also in Arrow et al.'s study, differences of supplemental injections between groups were not significant [13].

Data analysis of blood pressure, heart rate, and FLACC scale showed no significant differences between $4 \%$ articaine and $2 \%$ lidocaine $(P$ value $>0.05)$. The differences were neither statistically nor clinically significant. Although the mean score of WBFPS in the articaine group was significantly lower than lidocaine group $(P$ value $<0.05)$, the difference was less than one unit and this is not significant in clinical practices. Therefore, in the present study, similar volumes (both $1.8 \mathrm{ml}$ ) of $4 \%$ articaine and $2 \%$ lidocaine showed the same efficacy during maxillary primary molars extraction. However, with equal volumes of drugs, $4 \%$ articaine may contain twice as much active drug as equivalent volume of $2 \%$ lidocaine. This might be a reason for adequate diffusion of articaine into the palatal tissue. This aspect should be considered in future studies.

The intense pain of palatal injection can lead to children's misbehavior, so the authors suggest clinicians a single buccal infiltration of articaine and avoiding palatal infiltration at first. The pain sensation of palatal tissues must be assessed before the operation is initiated. In case of pain feeling in the palatal tissues, a palatal infiltration should be administered; otherwise, the palatal injection could be avoided.

As age increases, the bone porosity is decreased; thus, this clinical option can be practical for child patients; based on the present research, single buccal infiltration of articaine can be a practical clinical option during maxillary primary molar extraction in 6-9-year-old children.

However, broader age range of participants could give us more information about the exact age limit for administration of this technique. Also the present study did not determine the threshold volume required for the efficacy of this technique. Evaluation of these variables required a larger population of samples which was not possible during the COVID-19 pandemic.

Studies will be required to assess these aspects.

Root morphology has been an important risk indicator for complexity of tooth extraction or surgery [28], which can result in pain sensation or misbehavior of the pediatric 
patient. In the present study, teeth with at least one-third of the roots were extracted and patients who had teeth with resorbed roots were not enrolled into the trial. However, some levels of anatomic variations in the root morphology were inevitable.

\section{Conclusion}

Single buccal infiltration of $4 \%$ articaine can be administered as an alternative for conventional infiltration of $2 \%$ lidocaine to avoid fear or uncooperative behavior of the child patient. However, the assessment of palatal tissue anesthesia is quite necessary before the procedure is initiated. In case of failure in achieving palatal anesthesia, a palatal injection should be given.

Author contribution $\mathrm{ZB}$ and NM designed and wrote the paper; NM performed the interventions. Both authors read and approved the final manuscript.

Funding This research has been supported by Shahid Sadoughi University of Medical Sciences and Health Services.

Data availability Not applicable.

Code availability Not applicable.

\section{Declarations}

Ethics approval The study protocol was approved and supervised by the Ethics Committee of Shahid Sadoughi University of Medical Sciences in accordance with the declaration of Helsinki (DoH). The trial was registered at Iran Registration Clinical Trial Center with an online registration number: IRCT20191002044953N1.

Consent to participate Parents fulfilled the informed consent after receiving a complete description of the study process.

Consent for publication Written informed consent was obtained for publication of this study.

Conflict of interest The authors declare no competing interests.

\section{References}

1. Macpherson LM, Pine CM, Tochel C, Burnside G, Hosey MT, Adair P (2005) Factors influencing referral of children for dental extractions under general and local anaesthesia. Community Dent Health 22(4):282

2. Meechan JG (2000) Intra-oral topical anaesthetics: a review. J Dent 28(1):3-14

3. Ram D, Peretz B (2002) Administering local anaesthesia to paediatric dental patients-current status and prospects for the future. Int J Pediatr Dent 12(2):80-89
4. Baier K, Milgrom P, Russell S, Mancl L, Yoshida T (2004) Children's fear and behavior in private pediatric dentistry practices. Pediatr Dent 26(4):316-321

5. Tirupathi SP, Rajasekhar S (2020) Effect of precooling on pain during local anesthesia administration in children: a systematic review. Journal of Dental Anesthesia and Pain Medicine 20(3):119

6. Smolarek PD, Wambier LM, Siqueira Silva L, Chibinski AC (2020) Does computerized anaesthesia reduce pain during local anaesthesia in paediatric patients for dental treatment? A systematic review and meta-analysis. Int J Pediatr Dent 30(2):118-135

7. Gümüş H, Aydinbelge M (2020) Evaluation of effect of warm local anesthetics on pain perception during dental injections in children: a split-mouth randomized clinical trial. Clin Oral Invest 24(7):2315-2319

8. Malamed SF, GAGNON S, Leblanc D (2000) Efficacy of articaine: a new amide local anesthetic. The Journal of the American Dental Association. 131(5):635-42

9. Vähätalo K, Antila H, Lehtinen R (1993) Articaine and lidocaine for maxillary infiltration anesthesia. Anesthesia Progress. 40:114

10. Malamed SF, Gagnon S, Leblanc D (2001) Articaine hydrochloride: a study of the safety of a new amide local anesthetic. J Am Dent Assoc 132(2):177-185

11. Aps J, Badr N (2020) Narrative review: the evidence for neurotoxicity of dental local anesthetics. Journal of Dental Anesthesia and Pain Medicine 20(2):63

12. Alzahrani F, Duggal MS, Munyombwe T, Tahmassebi JF (2018) Anaesthetic efficacy of $4 \%$ articaine and $2 \%$ lidocaine for extraction and pulpotomy of mandibular primary molars: an equivalence parallel prospective randomized controlled trial. Int J Pediatr Dent 28(3):335-344

13. Arrow P (2012) A comparison of articaine $4 \%$ and lignocaine $2 \%$ in block and infiltration analgesia in children. Aust Dent J 57(3):325-333

14. Alinejhad D, Bahrololoomi Z, Navabazam A, Asayesh MA (2018) Comparison of visual analog scale scores in pain assessment during pulpotomy using different injection materials in children aged 6 to 8 and 8 to 10 years. The journal of contemporary dental practice 19(3):313-317

15. Malamed SF, Gagnon S, Leblanc D (2000) A comparison between articaine $\mathrm{HCl}$ and lidocaine $\mathrm{HCl}$ in pediatric dental patients. Pediatr Dent 22(4):307-311

16. Ram D, Amir E (2006) Comparison of articaine 4\% and lidocaine $2 \%$ in paediatric dental patients. Int J Pediatr Dent 16(4):252-256

17. Sandilya V, Andrade NN, Mathai PC, Aggarwal N, Sahu V, Nerurkar S (2019) A randomized control trial comparing buccal infiltration of $4 \%$ articaine with buccal and palatal infiltration of $2 \%$ lignocaine for the extraction of maxillary premolar teeth. Contemporary Clinical Dentistry 10(2):284

18 Sharma K, Sharma A, Aseri ML, Batta A, Singh V, Pilania D, Sharma YK (2014) Maxillary Posterior Teeth Removal Without Palatal Injection-Truth or Myth: A Dilemma for Oral Surgeons. Journal of clinical and diagnostic research: JCDR. 8(11):ZC01

19. Mittal M, Sharma S, Kumar A, Chopra R, Srivastava D (2015) Comparison of anesthetic efficacy of articaine and lidocaine during primary maxillary molar extractions in children. Pediatr Dent 37(7):520-524

20. Kolli NK, Nirmala SV, Nuvvula S (2017) The effectiveness of articaine and lidocaine single buccal infiltration versus conventional buccal and palatal injection using lidocaine during primary maxillary molar extraction: A randomized control trial. Anesth Essays Res 11(1):160

21. Tong HJ, Alzahrani FS, Sim YF, Tahmassebi JF, Duggal M (2018) Anaesthetic efficacy of articaine versus lidocaine in children's dentistry: a systematic review and meta-analysis. Int J Pediatr Dent 28(4):347-360 
22. McGuire DB (1992) Comprehensive and multidimensional assessment and measurement of pain. J Pain Symptom Manage 7(5):312-319

23. da Silva FC, Santos Thuler LC, de Leon-Casasola OA (2011) Validity and reliability of two pain assessment tools in Brazilian children and adolescents. J Clin Nurs 20(13-14):1842-1848

24. Vree TB, Gielen MJ (2005) Clinical pharmacology and the use of articaine for local and regional anaesthesia. Best Pract Res Clin Anaesthesiol 19(2):293-308

25. Tomlinson D, Von Baeyer CL, Stinson JN, Sung L (2010) A systematic review of faces scales for the self-report of pain intensity in children. Pediatrics 126(5):e1168-e1198

26. Ghadimi S, Shahrabi M, Khosravi Z, Behroozi R (2018) Efficacy of articaine infiltration versus lidocaine inferior alveolar nerve block for pulpotomy in mandibular primary second molars: a randomized clinical trial. Journal of Dental Research, Dental Clinics, Dental Prospects 12(2):97
27. Bataineh AB, Al-Sabri GA (2017) Extraction of maxillary teeth using articaine without a palatal injection: a comparison between the anterior and posterior regions of the maxilla. J Oral Maxillofac Surg 75(1):87-91

28. Benediktsdóttir IS, Wenzel A, Petersen JK, Hintze H (2004) Mandibular third molar removal: risk indicators for extended operation time, postoperative pain, and complications. Oral Surgery, Oral Medicine, Oral Pathology, Oral Radiology, and Endodontology 97(4):438-446

Publisher's note Springer Nature remains neutral with regard to jurisdictional claims in published maps and institutional affiliations. 